\title{
Ballistic Ureteroscopic Lithotripsy in Prepubertal Patients: A Feasible Option for Ureteral Stones
}

\author{
Paulo E. Fuganti, Silvio R. Pires, Renato O. Branco, Jose L. Porto \\ Section of Urology, Hospital Assunçao, Sao Bernardo do Campo, Sao Paulo, Brazil
}

\begin{abstract}
Objective: To evaluate the role of ballistic ureteroscopic lithotripsy in children with ureteral stones.

Materials and Methods: Children under 14 years with ureteral stones were treated with ureteroscopy in a 5-year period in our institution.

Results: Twenty-three procedures were performed in 20 children. Mean surgical time, age and stone size were $31 \mathrm{~min}$. (15 - 120min.), 11 years. (4-13 years), $5.3 \mathrm{~mm}(3-10 \mathrm{~mm})$ respectively. Three patients underwent two ballistic ureteroscopic lithotripsy each. There were 22 successful procedures (96\%) and a 100\% stone-free rate per patient. Complications (mucosal tear) occurred in 2 procedures $(8 \%)$ without extravasation of contrast media on retrograde pyelogram and their follow-up was uneventful.

Conclusion: Ureteroscopic ballistic lithotripsy is a feasible option for ureteral stones in prepubertal patients, with high stone-free rate and few complications.
\end{abstract}

Key words: ureteral calculi; child; ureteroscopy; complications

Int Braz J Urol. 2006; 32: 322-9

\section{INTRODUCTION}

Ureteral stones in children have been traditionally managed by extracorporeal shock wave lithotripsy (SWL), stenting and open surgery, while the smaller dimensions of the pediatric genitourinary system limited endourology. SWL replaced open surgery achieving high stone-free rates (1). Besides the need to eliminate stone fragments, re-treatment is eventually required (2) and differently from adults, SWL in children may require general anesthesia.

Endourological progress in the last decades changed the treatment of ureteral stones. Equipment miniaturization, surgical experience and new technologies on video-surgery promoted such evolution, rendering high success rates and few complications in adults $(3,4)$. Ureteroscopy has been applied to pediatric ureteral stones since $1988(5,6)$, but success rates and long term safety are still being addressed (7-9). Issues related to ureteral dilation, stenting, lithotripsy energy source and postoperative reflux are also not well defined, however, immediate ureteral clearing, stone resolution in a 24-hour postoperative hospital stay and fast recovering are appealing features of this method (7).

Since there are controversies about the optimal treatment for ureteral stones in children, we studied ballistic ureteroscopic lithotripsy treatment of ureteral calculi in the pediatric population focusing on success rates and complications. 


\section{MATERIALS AND METHODS}

Since 1999, all patients with ureteral stones who underwent ureteroscopy had a specific chart where preoperative, surgical and postoperative data were inserted in a computerized database. From July 1999 to June 2005, 1495 ureteroscopies for ureteral stones were included. Twenty-three $(1.5 \%)$ procedures were performed in children under 14 years old. Stones were diagnosed by ultrasound (US) and KUB. Intravenous pyelogram (IVP) or CT (computerized tomography) were indicated when diagnosis or stone location was not evident.

Two endoscopes were used: MRO7 (CirconACMI: length: $42 \mathrm{~cm}$, distal diameter: $7 \mathrm{~F}$ ) and 27400K (Karl-Storz: length $34 \mathrm{~cm}$, distal diameter 7.5F) semi-rigid ureterorenoscopes. An electrohydraulic lithotripter (Circon-ACMI: AEH-2A, probe 3F, length $120 \mathrm{~cm}$ ) and a pneumatic-ballistic lithotripter (Calculitus: maximal pressure 10 bar) were available, but all fragmentations were accomplished with the ballistic energy source with the aid of baskets (Cook: Helical stone extractor, size $3.2 \mathrm{~F}$, length $115 \mathrm{~cm}, 4$ wire basket).

A description of our technique is summarized below: after general anesthesia induction and prophylactic antibiotic (hospital: cefalotin sodium $50 \mathrm{mg} /$ $\mathrm{kg} / 24 \mathrm{~h}$, home: cefalexin 7 days), children were placed in lithotomy position and the endoscope was inserted into the urethra and the bladder. We did not employ a pediatric urethrocystoscope. Ureteral meatus was approached with the aid of the tip of a guidewire (Cook Urological: PTFE - shaft size 0.035", length $145 \mathrm{~cm}$, flexible tip $3 \mathrm{~cm}$; Bard: Hydro-Glide shaft size 0.035 ", length $145 \mathrm{~cm}$ ) followed by endoscope insertion. Dilation (Cook: fascial dilator set 6-18F, length $60 \mathrm{~cm}$ ) was not routinely used, unless severe edema prevented endoscopic access to the stone. The safety guidewire was advanced to renal pelvis only after the stone was visualized and when it could be advanced clearly between the stone and ureteral wall. Fragmentation was always tried when feasible while simple basketting was left for very small stones or its fragments. Retrograde pyelogram was routinely used at the end of the procedure to exclude ureteral perforations and false passages. Stents (Cook: double pig- tail stent set $4.7 \mathrm{~F}$ and $6 \mathrm{~F}$, length $26 \mathrm{~cm}$ ) were used in the presence of complications (4 weeks.) or severe edema ( 1 week.). A variable length from the vesical extremity was cut to adapt to child's height and the tip was tied to a 4.0 mononylon, exteriorized and adhered to penile/perineal skin. We did not use mononylon exteriorization when stent stay exceeded one week. Such patients and patients whose mononylon was displaced into the bladder required cystoscopy and stent retrieval with baskets.

Any residual ureteral stone was considered a failure. All children with intraoperative complications underwent IVP 3 months after surgery regardless of symptoms. All patients underwent US on follow-up (3 months after surgery), but only symptomatic patients or persistent hydronephrosis had an IVP. Routine postoperative cystourethrography, urinalysis or urine cultures were not performed.

\section{RESULTS}

Twenty-three procedures were performed in 20 children. Most of the children were boys (85\%) and two (10\%) of them had previous SWL. Mean surgical time, age and stone size were $31 \mathrm{~min}$. (15$120 \mathrm{~min}), 11$ years (4-13 years) $5 \mathrm{~mm} .(3-10 \mathrm{~mm})$ respectively (Table-1). There was one (4\%) failed ureteroscopy and a $100 \%$ ureteral stone-free rate per patient. Fifteen $(63 \%)$ procedures were stented. All patients were discharged home 24 hours after the procedure.

Three children underwent two procedures: patient \#5: bilateral ureteral stones treated at the same time; patient \#13: two episodes of impacted ureteral stones in the same year; patient \#6: failure of the first procedure due to intense edema of the ureteral orifice (stone not visualized), stented and successfully reapproached after one week.

We found mucosal tearing without extravasation of contrast media after two $(8 \%)$ procedures (\#2 and \#3): in procedure \#2 we had much difficulty to reach the stone due to its location (iliac vessels) and the stone could not be pulled to a more distal and amenable location to be fragmented. Despite these difficulties, ureterolithotripsy was carried out success- 
Table 1 - Patients characteristics and results of stone treatment.

\begin{tabular}{|c|c|c|c|c|c|c|c|c|c|c|c|}
\hline Procedure & Patient & Gender & $\begin{array}{c}\text { Age } \\
\text { (years) }\end{array}$ & $\begin{array}{c}\text { Previous } \\
\text { Treatment }\end{array}$ & $\begin{array}{l}\text { Stone Size } \\
\quad(\mathbf{m m} .)\end{array}$ & $\begin{array}{c}\text { Stone } \\
\text { Location }\end{array}$ & $\begin{array}{l}\text { Operative } \\
\text { Time (min.) }\end{array}$ & Result & Stent & Complication & $\begin{array}{l}\text { Follow } \\
\text { up (m) }\end{array}$ \\
\hline$\# 1$ & $\# 1$ & male & 12 & SWL & 8 & distal & 30 & success & yes & no & 8 \\
\hline$\# 2$ & $\# 2$ & male & 5 & no & 10 & iliac vessels & 120 & success & yes & mucosal tear & 47 \\
\hline$\# 3$ & $\# 3$ & male & 13 & no & 5 & distal & 20 & success & yes & mucosal tear & 10 \\
\hline \#4 & \#4 & female & 13 & no & 5 & distal & 30 & success & yes & no & 5 \\
\hline$\# 5$ & $\# 5$ & male & 13 & no & 7 & distal & 40 & success & no & no & - \\
\hline$\# 6$ & $\# 5$ & male & 13 & no & 7 & distal & & success & no & no & 13 \\
\hline$\# 7$ & $\# 6$ & male & 11 & no & 3 & distal & 50 & failure & yes & no & - \\
\hline$\# 8$ & $\# 6$ & male & 11 & no & 3 & distal & 30 & success & yes & no & 18 \\
\hline$\# 9$ & $\# 7$ & male & 7 & SWL & 6 & distal & 20 & success & yes & no & 13 \\
\hline$\# 10$ & $\# 8$ & male & 13 & no & 5 & distal & 30 & success & no & no & 7 \\
\hline \#11 & $\# 9$ & female & 13 & no & 5 & distal & 30 & success & no & no & 18 \\
\hline$\# 12$ & $\# 10$ & female & 12 & no & 4 & distal & 20 & success & yes & no & 26 \\
\hline$\# 13$ & $\# 11$ & male & 10 & no & 6 & distal & 20 & success & no & no & 15 \\
\hline$\# 14$ & $\# 12$ & male & 10 & no & 5 & distal & 40 & success & yes & no & 17 \\
\hline \#15 & $\# 13$ & female & 13 & no & 6 & distal & 30 & success & no & no & - \\
\hline$\# 16$ & $\# 13$ & female & 13 & no & 5 & distal & 15 & success & no & no & 12 \\
\hline$\# 17$ & $\# 14$ & female & 8 & no & 5 & distal & 15 & success & yes & no & 20 \\
\hline$\# 18$ & $\# 15$ & female & 12 & no & 4 & distal & 20 & success & yes & no & 6 \\
\hline$\# 19$ & \#16 & female & 13 & no & 4 & distal & 30 & success & no & no & 18 \\
\hline$\# 20$ & $\# 17$ & male & 11 & no & 4 & distal & 20 & success & yes & no & 14 \\
\hline$\# 21$ & $\# 18$ & male & 11 & no & 5 & proximal & 30 & success & yes & no & 11 \\
\hline$\# 22$ & $\# 19$ & male & 4 & no & 6 & proximal & 30 & success & yes & no & 4 \\
\hline$\# 23$ & $\# 20$ & male & 13 & no & 5 & distal & 15 & success & yes & no & 9 \\
\hline Mean & & & \multicolumn{2}{|c|}{$11(4-13)$} & \multicolumn{2}{|c|}{$5.3(3-10)$} & \multicolumn{2}{|c|}{$31(15-120)$} & & & \\
\hline Frequency & & & & $8 \%$ & & & & $96 \%$ & $63 \%$ & $8 \%$ & 14.5 \\
\hline
\end{tabular}

$S W L=$ extracorporeal shock wave lithotripsy; $m=$ months. 
fully in this location and retrograde pyelogram showed no extravasation. Stone in procedure \#3 showed an intense ureteral edema and was fragmented and retrieved with basket. Before stenting this patient, we could see a mucosal tear along the lateral ureteral wall. Contrast media injection showed a submucosal false passage not reaching retroperitoneal space. Both patients were asymptomatic on follow-up and IVP was unremarkable.

Moderate hydronephrosis persisted on follow-up in patient \#5, however his postoperative IVP did not show obstruction and he had symptoms for one year before ureteroscopy, suggesting sequela from long time obstruction. Patient \#18 had flank pain and underwent IVP, but no obstruction was found. Urine culture was negative and pain resolved spontaneously.

\section{COMMENTS}

We performed our first ureteroscopy in an adult patient in 1995. At this time, SWL was not available in our hospital and patients with ureteral calculi were sent to another institution. Such limitation helped us to increase our endourological experience while failures and complications felt to a minimal rate (4). After 1998, we then expanded ureteroscopic ballistic lithotripsy to children and their results remained similar to adults. Though we now have SWL, no children with ureterolithiasis in our institution have been treated with SWL.

Most ureteral stones sized less than $5 \mathrm{~mm}$ will be spontaneously eliminated in adults. Surgery is limited to $2 \%$ of cases while conservative treatment remains the best option for ureterolithiasis in children as in adults (10). Savage et al. (11) studied patients under 18 years (mean $=12$ years) who were treated for ureteral stones. Only $36 \%$ of 33 stones passed spontaneously and no stone greater than $3 \mathrm{~mm}$ was eliminated. Fifty five percent of stones sized less than $4 \mathrm{~mm}$ passed spontaneously. Thus, some children with ureteral stones will need active medical treatment.

SWL is the first line treatment for ureteral stones in children in some institutions. Muslumanoglu et al. achieved a $90.6 \%$ stone free rate for distal ure- teral stones smaller than $10 \mathrm{~mm}$ and Landau et al. reported a $100 \%$ stone free rate for lower ureteral stones. On the other side, these results are associated to a re-treatment rate of $30 \%$ and $51 \%$, respectively. Such inconvenience is avoidable with endoscopic treatment, as only 1 (4\%) of our children required a repeated procedure.

As reported in other series $(7,12,13)$, we could show that delicate handling of ureteroscopes and baskets allow stone treatment with few complications and high success rates in pediatric patients. Some aspects of our technique must be clarified as follows. The use of a pediatric urethrocystoscope is dispensable, as the ureteroscope also allows stone and stent retrieval. Inserting the guidewire into the ureter is a main step. We always try to advance it only when facing a space between the stone and the ureteral mucosa. This detail is paramount because we had adults whose ureteral submucosa was inadvertently penetrated at the level of the stone by the blinding advancement of the guidewire, which was only noticed after stone fragmentation. We do not advocate routine ureteral dilation and it was required in only one child (procedure \#7). The tip of the endoscopes allows easy penetration into the ureter. Different energy sources have been applied to pediatric ureteroscopic lithotripsy $(8,12,14)$. We applied electrohydraulic energy in a few adults, but it was replaced by the pneumatic-ballistic device. Our choice was due to its superior efficiency and precision to drive the impact to the exact point in the stone we want to fragment. Some authors also utilized ballistic energy in children with excellent results $(8,14-16)$. We always fragmented the entirely stone into minimal fragments. Since we had an ureteral avulsion in an adult patient, we prefer to extract only small fragments with special attention to the edematous ureteral mucosa surrounding the stone. We routinely perform retrograde pyelogram in children and adults. Visual inspection of a manipulated and inflamed ureteral wall is not reliable. We had adults whose ureteral perforation was only diagnosed by pyelography. As other authors $(7,9,16)$, we stented most of our patients and, maybe it was not necessary. However, the aspect of a pediatric manipulated ureter favored ureteral stenting. 
Most pediatric ureteroscopy series also show a stone-free rate above $90 \%$. We had one failure (procedure \#7) in an 11-year-old boy. Safety guidewire was advanced above the stone, but intense ureteral edema distal to the stone prevented ureteroscopic access. We tried ureteral dilation but ureteral mucosa was upward directed with the dilators, what could complicate into avulsion. A stent was left and another ureteroscopy was scheduled to next week. At this time, the edema resolved and the stone could be easily treated, but a stent was left again. Such failure was not related to a specific pediatric limitation, as many adults in our experience have been managed in the same way when severe ureteral edema is present. Al Busaidy et al. (12) also reported a similar occurrence in their study, but their resolution required open surgery. Satar et al. (15) reported one failure secondary to a proximal stone push-up. Most of our children had stones located in the distal ureter and we did not have stone migration. We had no partial fragmentation, which was related by some authors as a cause of failure $(9,12,17)$.

Even though perforations and ureteral lacerations have been described in children requiring open surgery (12), most complications may be managed endoscopically (13). We had two minor complications restricted to mucosal tear and managed with stent. No ureteral stenosis was detected on follow-up of these cases. Two cases underwent previous unsuccessful SWL in other institutions and managed with ureteroscopy. No complications or difficulties were observed. We did not perform cystourethrography in our patients because reflux rarely occurs, with minimal consequences $(7,8)$ and it is associated to intraoperative ureteral dilation (12). Urinary tract infection $(8,16-18)$ and hematuria with clots $(16,18)$ were already described, but they rarely occur. In this manner, few complications were reported in pediatric ureteroscopy.

\section{CONCLUSION}

Ballistic ureteroscopic lithotripsy is a feasible option for ureteral stones in children, with high stonefree rate and few complications.

\section{CONFLICT OF INTEREST}

None declared.

\section{REFERENCES}

1. Landau EH, Gofrit ON, Shapiro A, Meretyk S, Katz G, Shenfeld OZ, et al.: Extracorporeal shock wave lithotripsy is highly effective for ureteral calculi in children. J Urol. 2001; 165: 2316-9.

2. Muslumanoglu AY, Tefekli A, Sarilar O, Binbay M, Altunrende F, Ozkuvanci U: Extracorporeal shock wave lithotripsy as first line treatment alternative for urinary tract stones in children: a large scale retrospective analysis. J Urol. 2003; 170: 2405-8.

3. Harmon WJ, Sershon PD, Blute ML, Patterson DE, Segura JW: Ureteroscopy: current practice and longterm complications. J Urol. 1997; 157: 28-32.

4. Porto JL, Fuganti PE, Branco RO, Pires SR: Pneumatic ureteroscopic lithotripsy: report of 320 cases. J Endourol. 2000; 14 (suppl 1): A115.

5. Shepherd P, Thomas R, Harmon EP: Urolithiasis in children: innovations in management. J Urol. 1988; 140: 790-2.

6. Ritchey M, Patterson DE, Kelalis PP, Segura JW: A case of pediatric ureteroscopic lasertripsy. J Urol. 1988; 139: $1272-4$.

7. Thomas JC, DeMarco RT, Donohoe JM, Adams MC, Brock JW 3rd, Pope JC 4th: Pediatric ureteroscopic stone management. J Urol. 2005; 174: 1072-4.

8. Schuster TG, Russell KY, Bloom DA, Koo HP, Faerber GJ: Ureteroscopy for the treatment of urolithiasis in children. J Urol. 2002; 167: 1813-15; discussion 1815-6.

9. Minevich E, Defoor W, Reddy P, Nishinaka K, Wacksman J, Sheldon C, et al.: Ureteroscopy is safe and effective in prepubertal children. J Urol. 2005; 174 : 276-9; discussion 279.

10. Segura JW, Preminger GM, Assimos DG, Dretler SP, Kahn RI, Lingeman JE, et al.: Ureteral Stones Clinical Guidelines Panel summary report on the management of ureteral calculi. The American Urological Association. J Urol. 1997; 158: 1915-21.

11. Van Savage JG, Palanca LG, Andersen RD, Rao GS, Slaughenhoupt BL: Treatment of distal ureteral stones in children: similarities to the american urological association guidelines in adults. J Urol. 2000; 164: 1089-93.

12. al Busaidy SS, Prem AR, Medhat M: Paediatric ureteroscopy for ureteric calculi: a 4-year experience. Br J Urol. 1997; 80: 797-801. 
13. Raza A, Smith G, Moussa S, Tolley D: Ureteroscopy in the management of pediatric urinary tract calculi. J Endourol. 2005; 19: 151-8.

14. De Dominicis M, Matarazzo E, Capozza N, Collura G, Caione P: Retrograde ureteroscopy for distal ureteric stone removal in children. BJU Int. 2005; 95: 1049-52.

15. Satar N, Zeren S, Bayazit Y, Aridogan IA, Soyupak B, Tansug Z: Rigid ureteroscopy for the treatment of ureteral calculi in children. J Urol. 2004; 172: 298-300.
16. Bassiri A, Ahmadnia H, Darabi MR, Yonessi M: Transureteral lithotripsy in pediatric practice. J Endourol. 2002; 16: 257-60.

17. Wollin TA, Teichman JM, Rogenes VJ, Razvi HA, Denstedt JD, Grasso M: Holmium:YAG lithotripsy in children. J Urol. 1999; 162: 1717-20.

18. Shroff S, Watson GM: Experience with ureteroscopy in children. Br J Urol. 1995; 75: 395-400.

\author{
Correspondence address: \\ Dr. Paulo Emílio Fuganti \\ Rua Pará 984 / 1302 \\ Londrina, PR, 86015-560, Brazil \\ Fax: + 5543 3323-2860 \\ E-mail:paulo100urologia@hotmail.com
}

\section{EDITORIAL COMMENT}

In this study, Fuganti et al. report their experience on ureteroscopic lithotripsy in children by using ballistic and electrohydraulic energy sources with a high success rate. The authors correctly conclude that semirigid retrograde ureteroscopy is an effective and safe method for prepubertal children with ureteral stones. However, it is worth noting some points. Patient selection is controversial in ureteroscopic management of pediatric ureteral stones. In the literature, the ideal algorithm of ureteral stone management in children has not been reported with scientific analysis. Unfortunately, this study is failing to not answer this question. Nevertheless, it does let us know that the semirigid ureteroscopes and inflexible energy source probes that are used routinely in adults can be safely used in children. But, it is also very vital to point out that this series include only 4 children younger than 10 years of age and only 2 proximal ureter stones. Complication rate might be expected to go higher in younger age groups where flexible ureteroscopy is not available. Authors openheartedly admit their bias on ureteroscopy over shock wave lithotripsy for ureteral stones. We must emphasize that shockwave lithotripsy has been a very useful primary tool to fragment stones particulary in proximal ureter of even much younger kids. There are also some points need to be stressed in the technique of pediatric ureteroscopy. Although most steps are very similar to adult counterparts in ureteroscopy, the surgeon should be very aware of prominent psoas muscle and remarkable high incidence of anatomic variations due to either congenital anomalies or reconstructive surgeries. We generally start the ureteroscopy with a low pressure retrograde pyelography to check the ureteral anatomy since almost no intravenous urographies are ordered 
in children now. Intramural ureter dilatation has been strongly suggested by many pediatric urologists but there have been no proof that it might permanently jeopardize the ureterovesical junction but we faced with some problems associated with ureteral baloon dilatations. We have observed that hydrodistention of intramural ureter with a pressure bag or an arthroscopy irrigation set is even possible. Fragmenting the stone can be sometimes very frustrating in small and inflamed ureters. If we are using an energy source other than Ho:YAG laser, stone size and stone composition in our opinion is

\section{EDITORIAL COMMENT}

This is an interesting paper highlighting an ever-increasing use of ureteroscopy in the pediatric age group.

As worldwide experience increases in pediatric ureteroscopy and with technological advances and miniaturization of instruments, more urologists are using either rigid or flexible ureteroscopy as a first line treatment for ureteric stones.

The main alternative is extracorporeal shock wave lithotripsy (SWL) however; one of the major drawbacks for SWL is the rate of retreatments required and therefore for younger children usually under the age of 12 repeat general anesthetics. The authors group of patients have a mean age of 11 years with 12 out of the 23 being over 12 years old. Children over 12 years old generally can be considered for SWL without general anesthesia. All patients in this group however required general anesthesia with 3 patients requiring repeat procedures and therefore 2 anesthetics each. important. Our threshold of leaving ureteral stent and schedule a relook procedure is very low in cases with any difficulty in advancing the ureteroscope or following the ureteral lumen. We believe that a "failed" ureteroscopy is a better outcome than a "complicated" one. We tend to stent children with office removal dangler on as authors described, but we no longer cut the excess coiling tip of the stent because of very high rate of bladder spasms and occasional severe hematuria. We either use the appropriate stent length or let it coil inside the bladder.
Dr. Selcuk Yucel Associate Professor of Urology Akdeniz University School of Medicine Antalya, Turkey E-mail:drsyucel@yahoo.com
The mean stone size was $5.3 \mathrm{~mm}$. The authors used ballistic lithotripsy, which is infrequently used in children (more common electrohydraulic lithotripsy), and more recently Holmium lasertripsy becoming more popular. Bassiri et al. (1) used ballistic lithotripsy in 34/66 children although there overall stone free rate was $88 \%$ the stone free rate for ballistic lithotripsy on its own was not stated. In 4 patients, stones migrated into the kidney during ureterorenoscopy but once again, the report does not state what modality was being used in these cases. Previous reports in adults have described the risk of propulsion of stones back into the kidney with ballistic lithotripsy. This is one of the major drawbacks of this technology.

The authors state that fragmentation was always tried unless there was a small fragment, which could be removed with a basket. I feel that if the stone can be removed in one piece in a basket or with stone graspers without trauma to the ureter, this should be tried in preference to disintegration, as there is less 
risk to the ureter from trauma and less chance of retreatment. Stone fragments $4 \mathrm{~mm}$ or less should be considered for removal intact if at all possible.

The authors used stents in the presence of severe edema or complications. Fifteen of the patients required stents, which in general were exteriorized for ease of subsequent removal. I do feel that the use of stents should be minimized as they can cause distress to child when exteriorized and also if indwelling require either a local anesthetic removal or general anesthetic depending on the child. We have used ureteric catheters overnight in children with ureteric edema without complication and these are easily removed the following day.

I do think that the use of ureteroscopy should be limited to those with significant expertise and only be undertaken as a primary treatment modality if SWL is not readily available. Ureteroscopy is generally safe but there is still a risk of vesicoureteric reflux, ureteric stricture and urethral stricture in boys secondary to instrumentation. Long term follow up of children treated with ureteroscopy is still lacking.

The age of the child, anatomy, stone size and location, available treatment modalities as well as local expertise should all be considered before deciding on whether ureteroscopy or SWL is a more appropriate primary treatment modality for the individual child.

The authors however have clearly demonstrated that ballistic lithotripsy has a good success rate in treating pediatric ureteric stones and is safe.

\section{REFERENCE}

1. Bassiri A, Ahmadnia H, Darabi MR, Yonessi M: Transureteral lithotripsy in pediatric practice. J Endourol. 2002; 16: 257-60.

Dr. A Raza

The Scottish Lithotriptor Centre

Western General Hospital

Edinburgh, Scotland, UK

E-mail: araza36624@aol.com 